\title{
Efficient Reconfigurable Architecture for Pricing Exotic Options
}

\author{
PIETER FABRY and DAVID THOMAS, Imperial College London
}

\begin{abstract}
This paper presents a new method for Monte-Carlo (MC) option pricing using FPGAs, which uses a discretespace random-walk over a binomial lattice, rather than the continuous space-walks used by existing approaches. The underlying hypothesis is that the discrete-space walk will significantly reduce the area needed for each MC engine, and the resulting increase in parallelisation and raw performance outweighs any accuracy losses introduced by the discretisation. Experimental results support this hypothesis, showing that for a given MC simulation size, there is no significant loss in accuracy by using a discrete space model for the path-dependent exotic financial options. Analysis of the binomial simulation model shows that only limitedprecision fixed point arithmetic is needed, and also shows that pairs of MC kernels are able to share RAM resources. When using realistic constraints on pricing problems, it was found that the size of a discrete-space MC engine can be kept to 370 Flip-Flops and 233 LUTs, allowing up to 3000 variance-reduced MC cores in one FPGA. The combination of a highly parallelisable architecture and model-specific optimisations means that the binomial pricing technique allows for a $50 \times$ improvement in throughput compared to existing FPGA approaches, without any reduction in accuracy.
\end{abstract}

CCS Concepts: • Computer systems organization $\rightarrow$ Embedded systems; Redundancy; Robotics; -Networks $\rightarrow$ Network reliability;

General Terms: Design and Analysis, Algorithms, Performance

Additional Key Words and Phrases: Exotic Financial Options, Reconfigurable Hardware, Monte Carlo Optimizations, Control Variate

ACM Reference Format:

Pieter Fabry and David Thomas, 2016. Efficient Reconfigurable Architecture for Pricing Exotic Options ACM Trans. Embedd. Comput. Syst. 9, 4, Article 39 (March 2010), 22 pages.

DOI : 0000001.0000001

\section{INTRODUCTION}

Derivative markets have grown significantly over the last century, due to speculators and hedgers finding option trading more attractive than trading the underlying assets. The recent rise of High-Frequency and Algorithmic Trading means that traders must find under-priced options as fast as possible - wait too long, and the supply-demand model will restore the option to market equilibrium and remove any potential profits. Risk management also requires rapid option pricing, as high-frequency trading means that companies could quickly become over exposed due to the rapid-changes in prices that underly their option portfolios.

FPGA solutions have previously been proposed as a means of rapidly calculating the value of an option, particularly for Monte-Carlo pricing of exotic options with no closed-form solution. FPGAs can support many parallel Monte-Carlo simulators, but because the accuracy of Monte Carlo simulations is proportional to the square root of the number of simulations, pricing is still slow. Techniques such as control-variates can help improve accuracy, but the raw simulation rate (steps per second per resource) still limits the minimum pricing time.

This paper presents and evaluates a new method for performing Monte Carlo option pricing on FPGAs, which uses a discrete-time and discrete-space model for the underlying random walk, rather than the traditional discrete-time, continuous-space approach. These random-walks can be implemented as the flipping of a biased coin, which describes a path through a binomial tree. Coin flipping is very efficient in hardware, so each simulator is much smaller than a traditional continuous-space Gaussian simulator, resulting in an order of magnitude increase in raw simulation rate. This approach is quite general and can be applied to many options, and we demonstrate 
it for Asian, Look-back and barrier exotic options, while also applying control-variate techniques. The binomial implementation is compared against traditional continuoustime Brownian random walk for FPGA-based pricing, showing that for the three pathdependent exotic options investigated, the binomial random walk model provides $50 \mathrm{x}$ the performance, while maintaining the same accuracy.

Our contributions are:

- A new hardware-oriented approach for Monte Carlo pricing, which uses discrete-time and space models rather than the traditional discrete-time continuous-space model used in software.

- An analysis of the error properties of the binomial model, which explores the ratio between $\mathrm{K}$ (number of simulations) and $\mathrm{T}$ (steps per simulation) in a fixed $\mathrm{K} T$ budget.

- An optimized FPGA architecture for implementing vanilla Asian, Look-Back, and barrier Option Pricing using the binomial approach, which can also incorporate control-variates.

- An evaluation of the binomial versus traditional Brownian approach in a Xilinx UltraScale FPGAs, showing that the binomial approach provides 50x times the performance for the same accuracy.

The paper is divided into four main sections. The first section defines the background of pricing exotic options using a traditional Gaussian random walk model. Section two introduces the binomial model and shows it has similar error-to-simulation size as the Gaussian model for the three path dependent options investigated. Section three describes the FPGA hardware with justifications for binomial-specific hardware optimizations. The final sections then directly evaluate the results of the system.

\section{OPTION PRICING BACKGROUND}

Options are a type of financial derivative which gives the owner of the option the right but, not the obligation, to buy (Call Option) or sell (Put Option) an underlying asset (for example, a stock) at a predefined exercise (or strike) price $S_{E}$. The option contract will be upheld by both the buyer and seller of the option, so the price of an option should reflect the eventual expected profit from the option in order to avoid arbitrage opportunities.

Vanilla European options allow owners to exercise the option only at the expiration date $T$. If the underlying asset $S_{t}$ is higher than the strike price $S_{E}$ at time $T$, then the owner of a European Call option can profit by exercising the option and then immediately selling the underlying asset. Vanilla European options can be valued directly via the closed-form Black-Scholes equation due to their simplicity [Black and Scholes 1973].

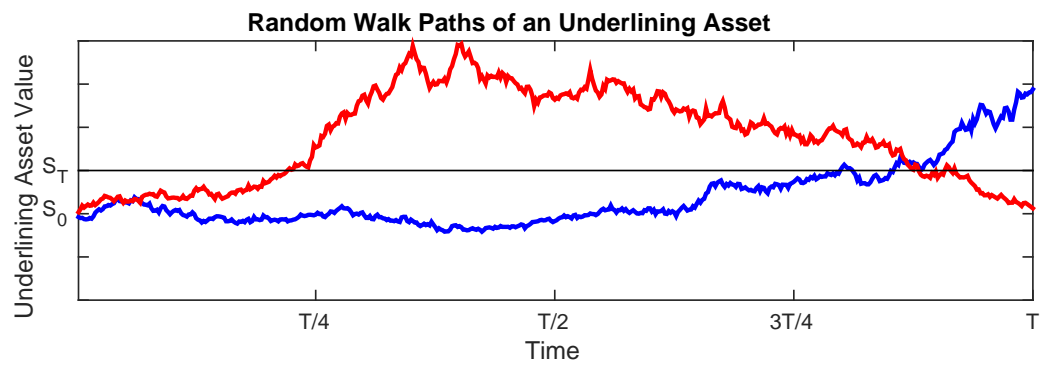

Fig. 1. An example of two random asset path walks. The value of the underlying is modelled according to the Black-Scholes assumption. The pay-off of a call option is the value of the option $S_{t}$ minus the strike price $S_{E}$ at time $T$. 
Consider Figure 1, which shows a hypothetical progression in value of a underlying asset across time. Two hypothetical random asset paths are shown via a red and blue line. If a European call option was bought for the red line, the option is profitable as the underlying asset $S$ at time $T$ is greater than the strike price of the option $S_{E}$. Therefore the owner of the call option can buy the underlining asset at price $S_{E}$ and immediately sell the asset for a profit of $S_{T}-S_{E}$. The blue line is unprofitable, as the underlining is less than the strike price $S_{E}$, so the owner will not exercise. The problem with vanilla European options is that, as in the case of the red line, the value of the option can be manipulated at the last minute to force arbitrage opportunities. For example, at time $T-\epsilon$, an investor could manipulate the market so that at time $T$ the value of the option is profitable.

Asian options are path-dependent financial options designed to combat manipulation of the market near the option expiry time. The payoff of the option is defined using the average value of the option throughout its lifetime, so manipulating the market near expiration day has minimal effect on the final value of the option. In Figure 1, the red line would remain unprofitable, since the average value is less than $S_{E}$, and investors cannot manipulate the options valuation over large time periods. Look-back options are another form of exotic option where the value of the option is a function of the minimum or maximum value achieved by the underlying, rather than the average as in Asian options. A third kind of exotic option is a Barrier option, where the value depends on whether or not the underlying price reaches a pre-set barrier level. Barrier options are designed to provide similar functionality to European options in normal market conditions, while being cheaper in extreme conditions where their value falls to zero. If the option is knock-in, the value of the option is non-zero only if the underlying price reaches the barrier level; if knock-out, the value of the option is zero once the value of the underlying reaches the barrier level.

While there are many closed-form [Camara 2009] and lattice-based pricing methods [Korn and Müller 2010] for various special-case options, in many cases exotic options cannot be directly priced, so we must fall back on Monte-Carlo simulation. Monte Carlo simulations use repeated random signals to approximate a numerical value that is computationally inefficient or impossible to calculate directly. Figure 1 can be seen as a very coarse Monte-Carlo simulation, with the red and blue lines representing two randomly generated paths, and the average of the two pay-offs is equivalent to a Monte Carlo simulation with simulation size $(K)$ equal to 2.

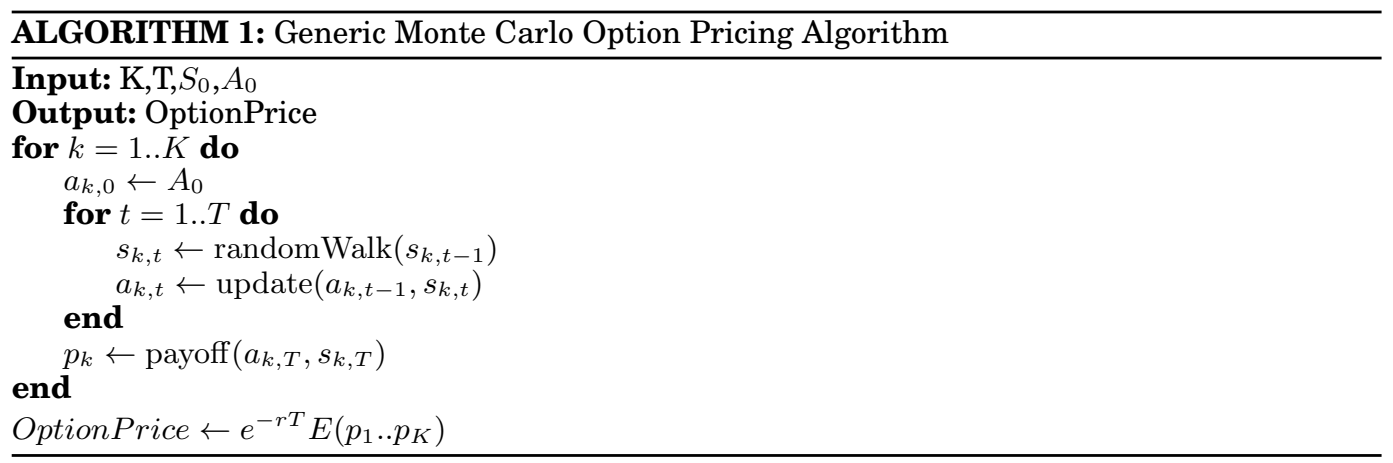

A generic description of the algorithm is described in Algorithm 1. The main loop of the algorithm iterates over the $K$ simulated paths. Each path is then stepped forwards for $T$ discrete time-steps to reach the expiry time, and the payoff is calculated. 
Without loss of generality, we will scale the expiry time of the option to match $T$, and take the time-step to be 1 . At each time-step the previous underlying $s_{k, t-1}$ is stepped forwards to the new value $s_{k, t}$, using a random walk function randomWalk. Under the Black-Scholes model, this step is log-normally distributed, with the mean and variance reflecting market interest rates and volatility. The discretized simulation of the random walk process (under the Euler-Maruyama scheme) is::

$$
\begin{aligned}
\operatorname{brownianWalk}(s) & =s e^{D+V W}, \quad \text { where } \\
D & =\left(r-\sigma^{2} / 2\right) \delta t \\
V & =\sigma \sqrt{\delta t} \\
W & \sim N(0,1)
\end{aligned}
$$

Here $D$ and $V$ are constants defined in terms of the implied volatility $(\sigma)$, interest rate $(r)$, and time-step $(\delta t)$. In this paper we assume that the option parameters have been scaled to give $\delta t=1$.

Once the random walk has been stepped, the option state must also be updated. For exotic options the option price depends on the path taken by the underlying, so this must be folded into the option state using update $(a, s)$. The definitions of this function for our three example options are given in Table I.

Table I. The Update Functions for the Options Investigated

\begin{tabular}{|l|l|l|l|}
\hline Exotic Option Function & Asian & Look-back & barrier \\
\hline update $(a, s)$ & $a_{t}=a_{t-1}+s_{t}$ & $a_{t}=\max \left(a_{t-1}, s_{t}\right)$ & $a_{t}= \begin{cases}1 & s_{t}>B \\
a_{t-1} & \text { otherwise }\end{cases}$ \\
\hline payoff $\left(a_{T}, s_{T}\right)$ & $p=\max \left(0, \frac{a_{T}}{T+1}-s_{T}\right)$ & $p=\max \left(0, a_{T}-s_{T}\right)$ & $p=a_{t} \max \left(0, s_{T}-S_{E}\right)$ \\
\hline
\end{tabular}

In general the variance of the result, which is essentially the accuracy of the option price, will decrease as $O(1 / \sqrt{K})$. This means that we must quadruple $\mathrm{K}$ in order to double the accuracy. One approach to reduce the time taken is to parallelise over the $\mathrm{K}$ simulations. This technique works well in any parallel platform, particularly FPGAs and GPUs, where it is very efficient to run multiple simulators. However, a substantial increase in parallelism is needed to get a moderate reduction in accuracy.

The time-to-result using the Monte Carlo approach is also made faster through variance reduction techniques [Echeverria et al. 2008]. One relevant technique is to use a control variate variable $c$, calculated from the same path used to generate $p$, the valuation of the option. If we choose $c$ such that a closed form solution True $e^{c}($.$) already$ exists, it is possible to track the average of $E(p-c)$, then estimate the option prices as $p_{c v} \approx E(p-c)+\operatorname{True}^{c}($.$) . Equation 5$ shows how the variance of $p$ can be reduced according to the correlation and variance of the underlying control variate $c$ :

$$
\operatorname{Var}\left(p_{c v}\right)=\operatorname{Var}(p)-\operatorname{Cov}(c, p)^{2} / \operatorname{Var}(c)
$$

Choosing a control variate with high correlation $(\operatorname{Cov}(c, p))$ to the desired option reduces the variance of the Monte Carlo simulation, and therefore significantly reduces the number of Monte Carlo simulations required. Algorithm 2, adapted from [Anson et al. 2010], highlights the adjustments required to implement a control variate algorithm on top of the basic Monte Carlo already seen in Algorithm 1. 


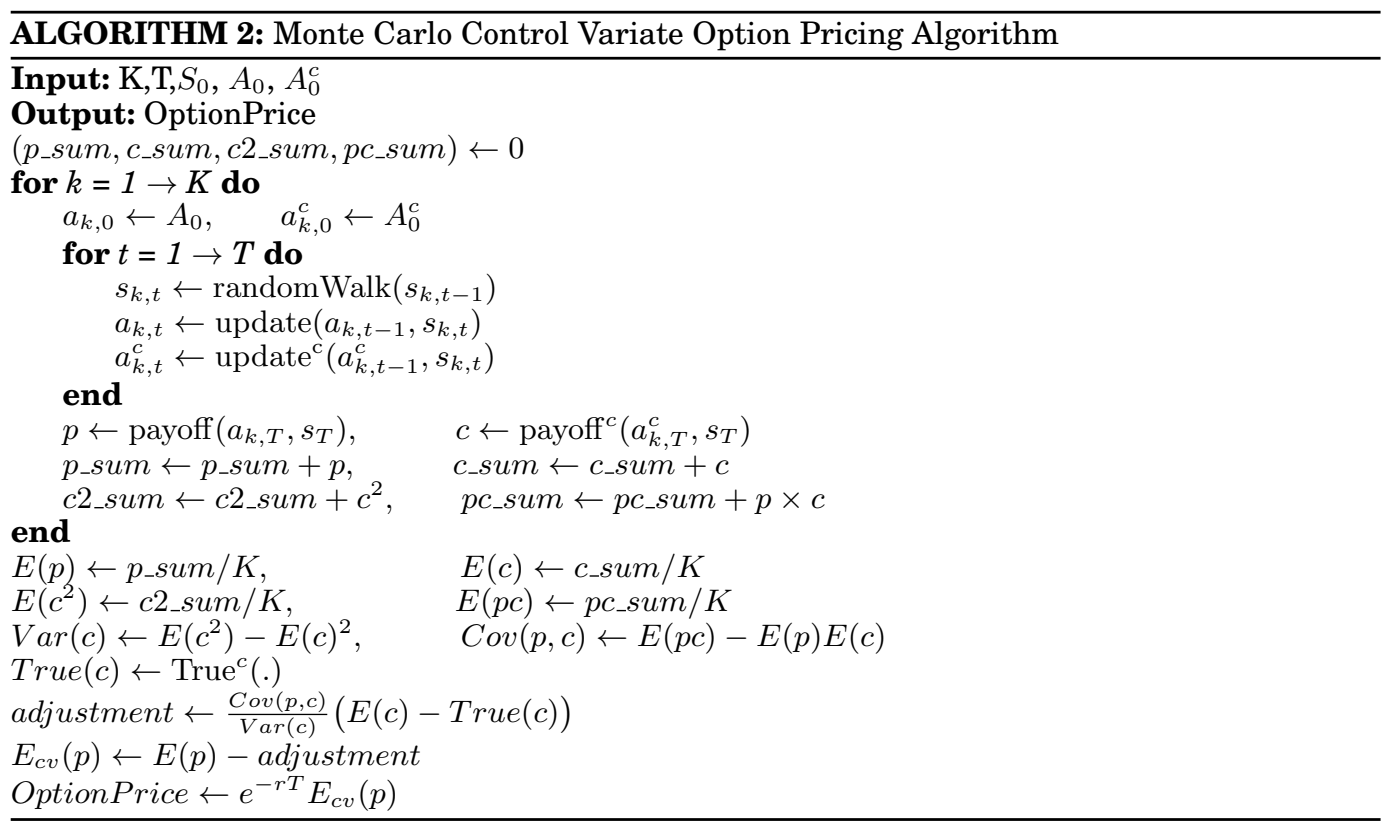

\section{RELATED WORK}

Monte Carlo option pricing techniques have been used since 1977 [Boyle 1977], with many modern Monte Carlo pricing schemes still using discrete-time continuous-space versions of the Gaussian random walks which underpin the Black-Scholes equations. More recently there have been moves to support more complex distributions, such as the Heston model [Heston 1993], which augments the random walk to support timevarying volatility and can better model market conditions. These more complex models are only possible due to increasing CPU performance, and in many Monte-Carlo simulations the Black-Scholes model is still used in order to limit execution time.

Due to the parallelism exposed in Monte-Carlo, there are a number of examples of FPGA architectures for the discrete-time continuous-space model of exotic option pricing [Tse et al. 2010; de Schryver et al. 2013; Sridharan et al. 2012; De Schryver et al. 2011; Zhang et al. 2005], using kernel-based architectures where the option logic can be extendible to other exotic options. The core idea is to create random walk kernels which evaluate many random walk paths in parallel on FPGAs. This solution is scalable and fast, with its time to result dependent on the permissible error and the number of kernels placed on the FPGA. The more kernels that can fit on the FPGA, the more simulations can be executed in parallel, so performance can be increased by decreasing the size of the kernels. However, the continuous-space approach requires random sampling from a Gaussian or log-normal distribution, and the accumulation and stepping require floating-point operations, so FPGA resources are the limiting factor in the concurrency of the design.

Control Variate techniques on FPGA have also been proposed [Anson et al. 2010; Boughamoura and Trabelsi 2011], using many parallel continuous-space simulators. One approach uses a European option as the control variate $c$, such that True $(c)$ is the Black-Scholes valuation. Since the degree by which variance is reduced is related to the correlation between $p$ and $c$, a control variate which has intrinsic path-dependence (unlike European options) will likely have a higher correlation. 
Multi-level Monte Carlo (MLMC) ([Giles 2015; 2008; de Schryver et al. 2013]) is another technique whereby the accuracy of the result from Monte Carlo simulation can be improved without increasing computational time. The idea is to introduce multiple levels of resolution in a Monte Carlo simulation, so rather than a single value of $T$ and $K$, we choose a number of time-resolutions $T_{1} . . T_{l}$, and run $K_{1} . . K_{l}$ simulations for each level. It is then possible to combine the results in such a way as to reduce the variance of the paths. This technique still requires a forward-moving Monte Carlo asset estimation, which may be based on a standard walk such as Brownian or Heston. We do not consider MLMC here as it is a general variance reduction technique; if the approach used here is used to increase the fundamental stepping performance, the MLMC can be applied on top using software, which is left as future work..

\section{DISCRETE-SPACE MONTE CARLO}

Existing FPGA simulation techniques are based on the idea of continuous-space random-walks, so $s_{t}$ is a continuous variable, and randomWalk $(s)$ attempts to sample from a continuous distribution. As we saw in Equation 4, this requires:

- Sampling from the Gaussian distribution;

- Calculating an exponential function;

- Performing multiple additions and subtractions.

These operations result in Monte-Carlo simulation kernels which are comparatively large, limiting us to 10-100 parallel kernels in modern FPGAs.

Here we propose that it makes more sense to rethink the simulation in terms of operations that an FPGA is particularly good at, in order to minimise the size of each kernel. The strengths we identify are:

- Uniform-random numbers : FPGAs are very efficient at generating random bits, requiring only $w$ LUTs to generate a $w$ bit random number.

- Look-ups in small tables : while FPGAs have limited on-board RAM, if look-up tables fit into block-RAMs the FPGA can support 1000s of lookups per cycle.

- Fixed-point addition: addition or comparison of $w$ bit integers only requires $w$ LUTs. For this paper, we used $w=32,8$ integer bits, 24 fractional bits.

To exploit these strengths, we suggest that instead of using a continuous-space random walk, we restrict ourselves to a discrete-space random walk. So rather than the full Gaussian step, we calculate $s_{t}$ as:

$$
s_{t}=\left\{\begin{array}{ll}
s_{t-1} u, & \text { if } P_{u} \leq r_{t} \\
s_{t-1} d, & \text { otherwise }
\end{array}, \quad \text { where } r_{t} \sim U(0,1)\right.
$$

So at each time-step we toss a biased coin, and then move up by a factor $u$ with probability $P_{u}$, or move down by a factor $d$ with probability $1-P_{u}$. Each step represents a sample from the log-Bernoulli distribution, so the overall effect is that $S_{T}$ will be the product of $T$ independent log-Bernoulli samples, so $S_{T}$ is also a sample from the $\log$-binomial distribution $S_{T} / S_{0} \sim \exp \left(\operatorname{Bin}\left(T, P_{u}\right)\right)$. As $T \rightarrow \infty$, the Central Limit Theorem means that this distribution must converge on the log-normal distribution, for the same reason that the binomial distribution converges on the normal distribution.

This convergence is show graphically in Figure 2, with $T$ varying from $T=4$ on the left through to $T=256$ on the right. The bottom row shows continuous-time Gaussian walks, so we can see that while the bottom-left occupies many points in space over the different paths, it is still coarse in time. The top-left shows the discrete-time equivalent, where the discreteness in both space and time is highly-visible, so for $\mathrm{T}=4$ the discrete approach does not approximate the desired distribution. However, for $\mathrm{T}=256$ 

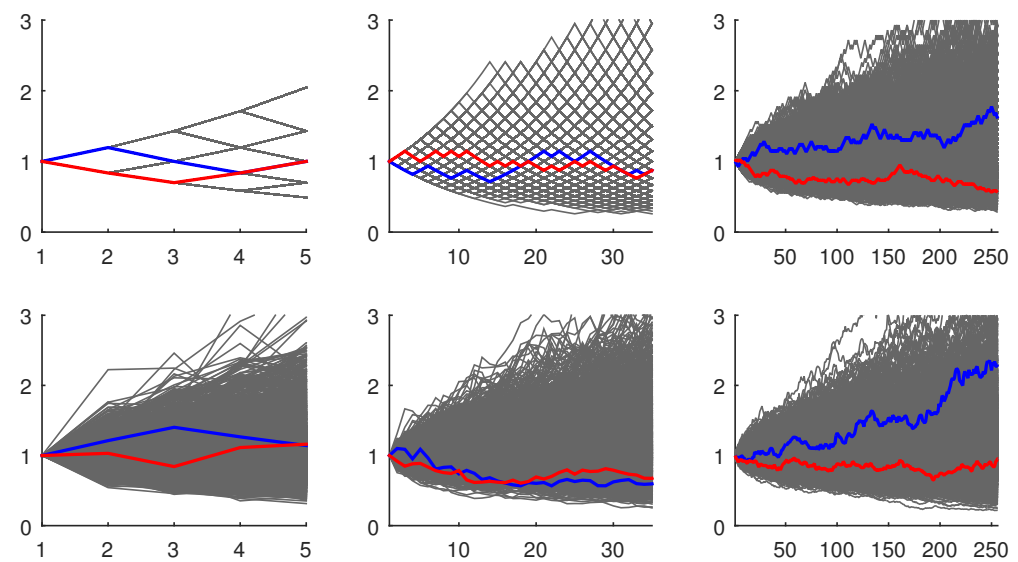

Fig. 2. The binomial walk follows discrete values, while a Gaussian walk takes a continuous range of values at each step. The red and blue lines indicate possible random walks.

on the right-hand side, the individual discrete and continuous walks look very similar (compare the red and blue lines), and also appear to have similar gross statistical properties (the shape of the grey mass).

The discrete walk requires us to find values of $P_{u}, u$, and $d$ that will approximate the desired Gaussian path. The main criterion needed is that the discrete and Gaussian should match in their first and second moments (mean and variance). If this condition is met, then the Central Limit Theorem guarantees eventual convergence in the limit. Here we borrow these parameters from the world of lattice pricing, which uses deterministic backwards induction in order to price options, rather than the stochastic forwards method used here.

A common method used in the Cox-Ross-Rubinstein (CRR) method [Cox et al. 1979], which prices options on a binomial lattice. In the binomial lattice, each price at $t-1$ is formed from a blended weight of the prices at $t$, which also relies on moment matching a binomial distribution. Under the CRR model $u d$, and $P_{u}$ can be calculated as:

$$
u=e^{\sigma \sqrt{\delta t}} \quad d=e^{-\sigma \sqrt{\delta t}}=u^{-1} \quad P_{u}=\frac{e^{r \delta t}-d}{u-d}
$$

While the CRR lattice is used for pricing American options, it does not handle pathdependent exotic because there is no concept of a 'path' in its evaluation.

The CRR lattice has the property that $u=1 / d$ which makes it a recombining tree, so an up-step followed by a down-step results in the same value. The recombining property can be seen visibly in Figure2, where it is responsible for the clear horizontal regularity seen in the first two discrete examples. Each time step $s_{t}$ can be expressed in the form:

$$
s_{t}=S_{0} u^{x}, \quad \text { where } \quad x=\sum_{j=1}^{t} \begin{cases}+1, & \text { if } P_{u} \leq r_{j} \\ -1, & \text { otherwise }\end{cases}
$$

This allows for another FPGA optimisation, which is to replace the multiplication with a table-lookup containing $L U T[x]=S_{0} u^{x}$. The current spatial level then becomes a simple integer counter, and hopefully the LUT can be small enough to store in one or more block-RAMs. The LUT contents only change when option parameters change, so 
Table II. The Random Walk Functions

\begin{tabular}{|c|c|c|c|}
\hline Random Walk Model & Gaussian & Binomial (Multiplicative) & Binomial (Indexed) \\
\hline $\operatorname{randomWalk}(S)$ & $\begin{aligned} w & \sim N(0,1) \\
s_{t+1} & =s_{t} e^{D+V w}\end{aligned}$ & $s_{t}= \begin{cases}s_{t-1} u & \text { if } P_{u} \leq \sim U(0,1) \\
s_{t-1} d & \text { otherwise }\end{cases}$ & $\begin{array}{c}x_{t}=\left\{\begin{array}{cl}x_{t-1}+1 & \text { if } P_{u} \leq \sim U(0,1) \\
x_{t-1}-1 \quad \text { otherwise }\end{array}\right. \\
s_{t}=L U T\left[x_{t}\right]\end{array}$ \\
\hline
\end{tabular}

all the LUTs on the chip can be initialised with the same entries just before simulation starts.

Table II summarises the Gaussian random walk and two variants of the binomial walk, which can all be plugged into Algorithm 1 or Algorithm 2. Due to the simplicity of the indexed walk over the LUT, we will not consider the multiplicative binomial walk any further here - while it may have advantages in other architectures, in an FPGA we found the need to manage rounding and range mean that DSP blocks became a constraint much faster than block-RAMs As we will show in Section 6, the indexed binomial walk can be further optimised for FPGA resources, resulting in the very high level of parallelism shown in Section7. However, we must first show that the binomial model retains sufficient accuracy, despite its highly discrete nature.

While asymptotically it is known that the binomial and Gaussian random walk models converge, for low step sizes it is clear from Figure 2 that the probability distributions are not the same, so we need to investigate whether the binomial model converges fast enough to be useful. Because the rate of convergence for Monte Carlo solutions is inversely proportional to the square root of the number of simulations done, a more accurate system will require the square of the number of simulation. Therefore, even if the binomial model can be made, say, $10 \times$ smaller than the Gaussian kernel and is only half as accurate, for higher accuracy models the Gaussian kernel will eventually still be superior.

\section{BALANCING STEPS VS PATH COUNT}

As identified in Algorithm 2, both the time to result and the accuracy of the result is a function of the iterations $(K)$ and the tree size $(T)$ of the model. Reducing either $K$ or $T$ will produce an answer sooner, at the cost of accuracy, while increasing $K$ or $T$ will improve the accuracy but require more time to compute. However, it isn't clear how to choose $K$ and $T$ in order to optimise error versus time. In order to explore this behaviour, we introduce the notion of the KT space, which maps each $(K, T)$ point to the mean-square error achieved for that combination. While the exact relationship between KT and error is unknown, we can empirically measure the KT space. The time taken to compute the Monte Carlo simulation is known to be $\mathbb{O}(K T)$, and can be exactly calculated in cycles for an FPGA implementation. So given a time-budget $K T$, we can use the empirical KT space to identify a good balance between $K$ and $T$ that will minimise the error.

The mean square error $\left(E_{K T}\right)$ of a Monte Carlo simulation can be calculated as:

$$
E_{K T}=\frac{1}{M} \sqrt{\sum_{m=1}^{M}\left(P_{K T, m}-T_{r}\right)^{2}}
$$

where $P_{K T, 1} . . P_{K T, M}$ are the results from $M$ Monte Carlo simulations at a size $K T$, and $T_{r}$ is the "true" value of an option value option's value. Due to the lack of closed-form exotic option pricers, we calculate $T_{r}$ via a Monte Carlo simulation with $K T=10^{10}$, and the optimal KT ratio is chosen by selecting the best ratio of a geometric Asian 
walk compared to the true value of the geometric Asian option. ${ }^{1}$ We estimated the KT space by choosing $M=30$, and then exploring the different $K T$ combinations. For our experiments we chose $S_{0}=50, \sigma=0.4, r=0.12$, and scaled the parameters to give an expiry time of 1 . For Asian and barrier options $S_{E}=52$ with the knock-out barrier set at 70, while for look-back options $S_{E}=70$. These parameters were chosen to ensure the results are of the same approximate value for better graphical comparison.

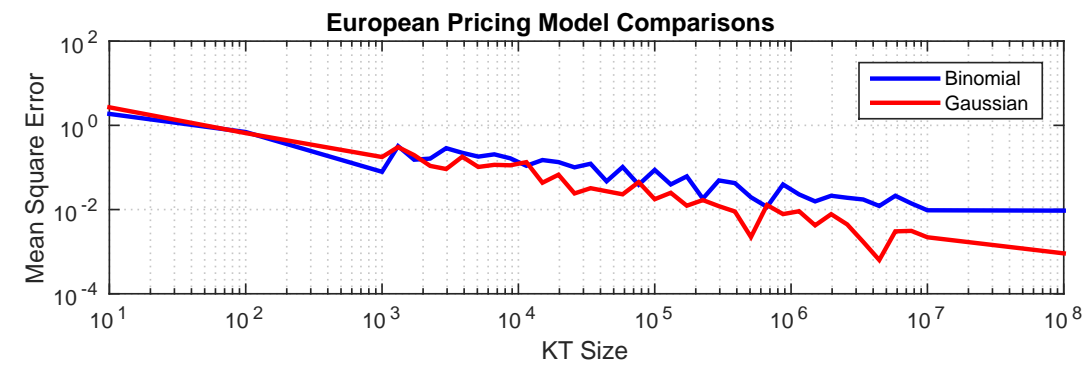

Fig. 3. The minimum error vs KT size of the binomial and Gaussian random walks pricing a European Option

We first consider the binomial vs Gaussian approach for European options - these are not path-dependent, so only $S_{T}$ matters, and we would expect the Gaussian approach to be more effective. Figure 3 shows the error against total $K T$, using the optimal KT tradeoff for each point, confirming that the binomial model is inappropriate for non-path dependent options. For a given KT size, the binomial model is less accurate than the Gaussian underlying model for pricing a European Option, and by a KT size of $10^{8}$ there is almost an order of magnitude difference in the achieved accuracy. The intuition here is that the binomial model is having to split its time budget between $T$ in order to improve the normal distribution at expiry time, and $K$, which reduces overall variance. In contrast, the Gaussian approach can simply take $T=1$ and $K=K T$, concentrating all of the effort on just the final time step. The x-scales for these graphs are the product of $\mathrm{K}$ and $\mathrm{T}$, where the Mean Square Error presented is the smallest error found via Monte Carlo simulation a given KT size (IE the mean square error of the best KT combination).
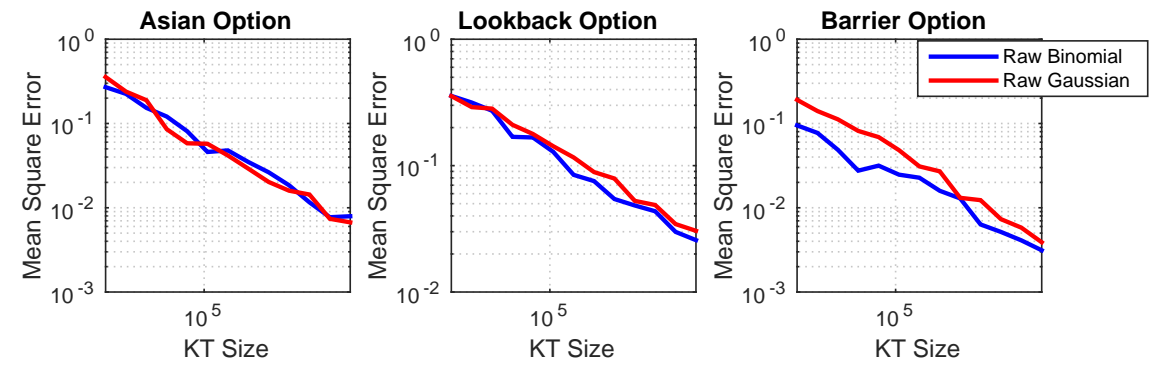

Fig. 4. The minimum error vs KT size of the binomial and Gaussian random walks

The same test was then performed on path-dependent options - lookback, arithmetic Asian, and barrier - using the "raw" algorithm 1 and no control variates. Figure 4

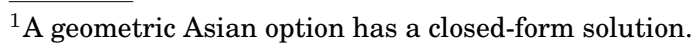


shows that increasing the KT space broadly reduces the error according to the centrallimit theorem, with the errors following a linear trend in log-log space. For the Asian and lookback option it can be seen that both the Gaussian and binomial models overlap and follow the same gradient, and so can be used interchangeably. However, this is not the case for the barrier option, where the binomial method has a small but consistent advantage in error. This is because the barrier option can knock out part way through the walk, and the binomial method is better a consistently exploring these knock-out points, effectively providing a simple form of variance-reduction.

Figure 5 explores how the optimal number of steps $(T)$ increases with the $K T$ size. For both the lookback and Asian option, there is little difference between Gaussian and Binomial models, with the increase in $T$ happening at about the same rate. This reflects the similar error convergence seen in Figure 4, where the binomial and Gaussian appear to be statistically equivalent. However, we see that the optimal number of steps for the barrier option varies widely between binomial and Gaussian, with the Gaussian consistently requiring more effort placed on $T$. As a consequence, the Gaussian method cannot perform as many paths for a given total $K T$ effort, so the difference in steps for the barrier option in Figure 5 explains the difference in error seen for the barrier in Figure 4.
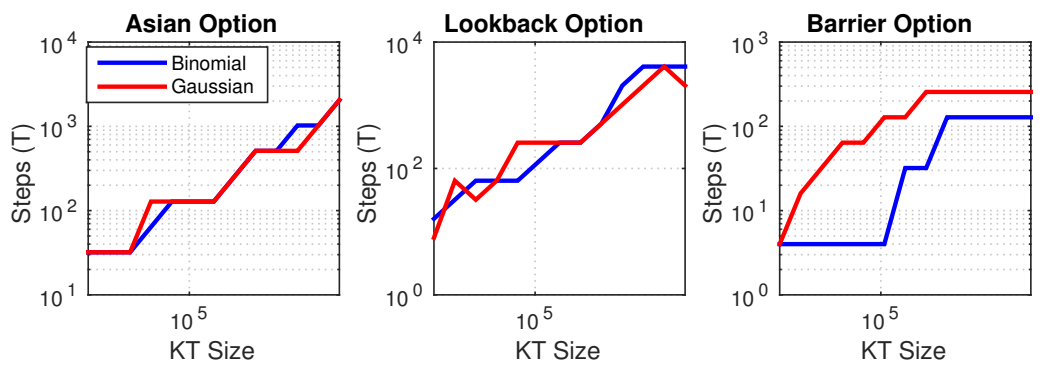

Fig. 5. The number of steps taken for the optimal result of a given KT Size

We will now explore the effect of control-variates on the binomial and Gaussian convergence, as any practical contemporary implementation of the Gaussian method will already use control-variates. Figure 6 shows the result of applying Algorithm 2 using Gaussian walks, using three choices of control-variates: raw (no control variate), European, and geometric Asian. European control variates have previously been used in hardware [Tse et al. 2010], due to the simplicity of the resulting hardware, but are only weakly correlated to path dependent options. As a result, the European controlvariate offers limited benefits in error, especially for the barrier option. In this work we also consider geometric Asian options, as they are better correlated with heavily path dependent options [Fu et al. 1999]. The results show that, even if the hardware is a little more complicated, the benefits in error are substantial, allowing the same error for an order of magnitude smaller KT space.

Control variates are already known to work well for Gaussian walks, but we must again ask the question of whether they still hold for the highly discretised binomial walks. Figure 7 repeats the previous experiment with the binomial walk, and shows that:

- The control variate technique still works well for binomial walks.

— The geometric Asian is still consistently the superior control variate. 

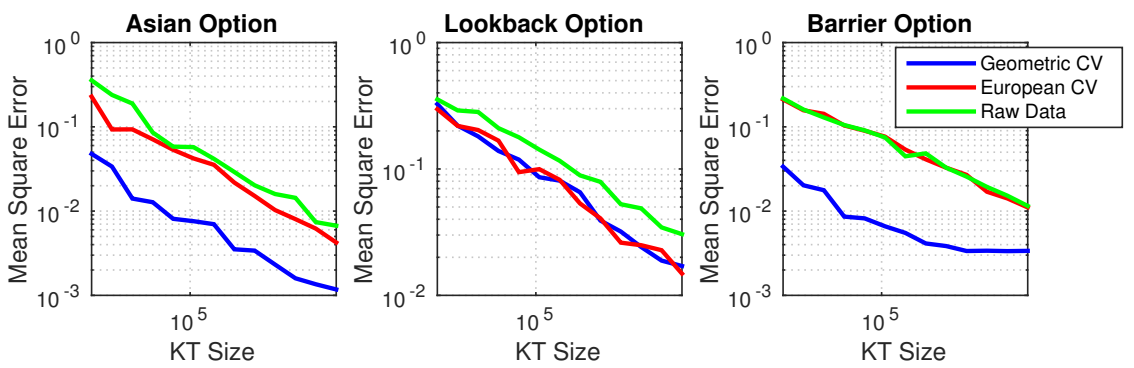

Fig. 6. Comparisons of control variate methods for Gaussian Walks.

However, we see two interesting differences in behaviour as well. First, the European CV is not as effective for the lookback option, even though it worked very well for the Gaussian version. This appears to be due to the earlier results in Figure 3, which showed that the binomial method is not very inaccurate at evaluating European options. Second, the European CV is more effective for the barrier option at lower $K T$, which appears to be due to the difference in $T$ seen in Figure 5; the binomial method is able to use much larger $K$ for small $K T$ values which reduces error, but for larger $K T$ values the binomial and Gaussian use similar $T$.
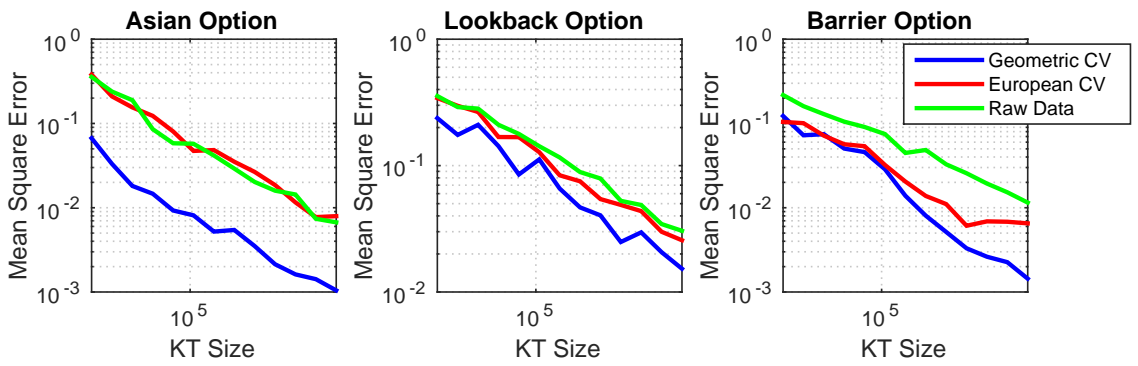

Fig. 7. Comparisons of control variate methods for Binomial Walks.

Given the geometric Asian is the best control variate for all three options, we repeat the earlier experiments to show the relationship between error and total $K T$ in Figure 8, and the optimal $T$ selected in Figure 9. We find that the Gaussian and binomial walks exhibit the same error vs $K T$ performance for all but the barrier option, and in that case the binomial performs better for larger $K T$. As a consequence, we can conclude the binomial model in hardware will be as accurate as Gaussian model for the same $K T$ size. So if we are able to put many more parallel binomial evaluators into hardware, that increase will go directly to improving error, without having to overcome any intrinsic inaccuracies of the binomial method first.

The experiments in this section have considered a static $K: T$ balance for a given $K T$ size, using experiments to choose good balances for each option type. Another approach would be to use a more dynamic approach, which tries to combine results from a number of runs with different $T$ values. A robust method for doing this is the multilevel Monte-Carlo approach [Giles 2008], which tries to minimise total variance by optimising both $K$ and $T$ at run-time. While we have chosen a static approach here, there is nothing to suggest that a multi-level approach could not be used instead, and this will be covered in future work. 

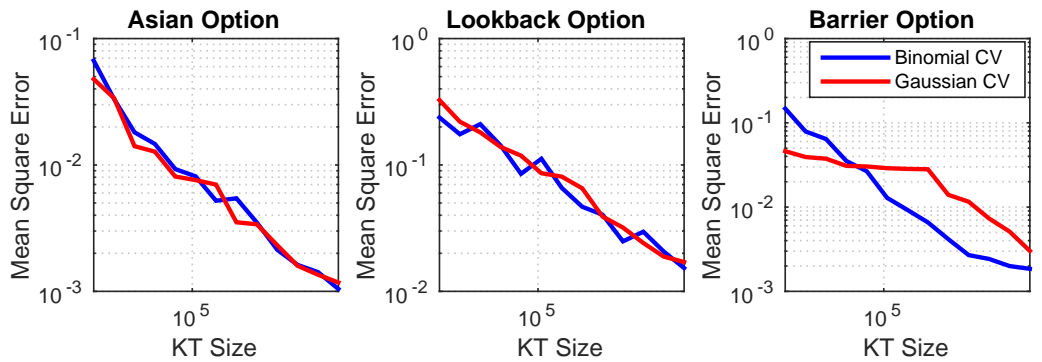

Fig. 8. The minimum error vs KT space of the binomial and Gaussian random walks
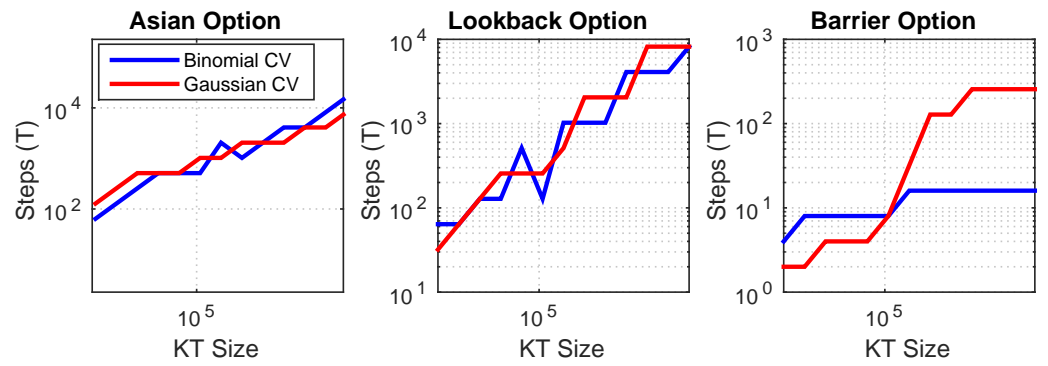

Fig. 9. The number of steps taken for the optimal CV result of a given KT Size

\section{FPGA ARCHITECTURE}

We will now discuss our FPGA architecture, which allows an FPGA to be filled with many small and fast binomial random walk kernels. The more kernels that can be placed in parallel on FPGA, the more iterations of a Monte Carlo path can be performed concurrently, with the time taken to price an option inversely proportional to the number of kernels $(N): O\left(\frac{K T}{N}\right)$. So if we can achieve a much larger $N$ using binomial kernels than Gaussian kernels, then the results from the previous section show than we will achieve a much lower error in the same amount of time.

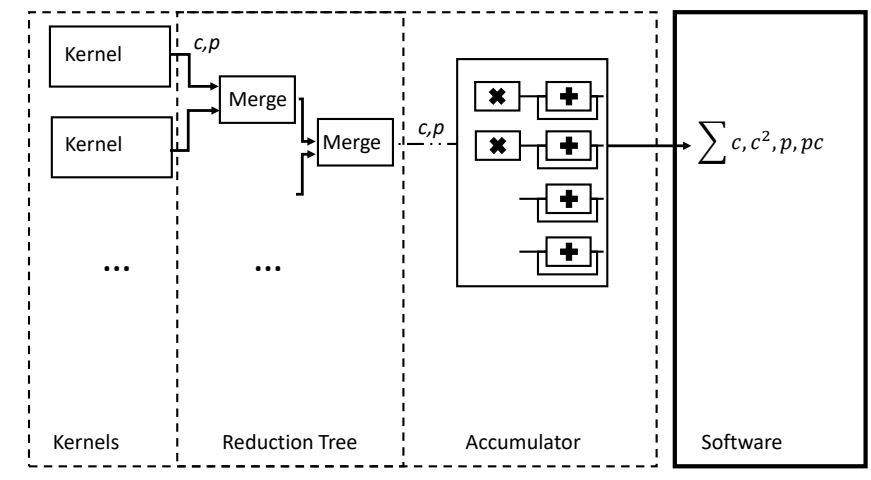

Fig. 10. Top-level block diagram of the FPGA pricing architecture

The overall architecture of our approach is shown in Figure 10, and contains four components: 
-Kernels : Each kernel is an independent Monte-Carlo path simulator, which produces pairs of option and control-variate payoffs.

- Reduction Tree : The output of hundreds or thousands of kernels is interleaved in time using a tree, producing one single stream of option payoff pairs.

- Accumulator : The stream of option pairs is processed and accumulated, in order to track a tuple of running statistics of the payoffs and correlations.

- Software : The tuple of raw statistics is post-processed in $\mathrm{O}(1)$ time to produce the final variance-reduced option value.

The expectation is that the vast majority of the FPGA will be taken up with kernels, in order to complete as many paths steps per second as possible. In contrast, only a small number of accumulators are needed (usually just one), as the kernels produce one value every $T$ cycles, so one accumulator can support $T$ kernels.

\subsection{Kernel}

Each kernel outputs $(p, c)$, which is a pair of the option payout $p$ and the control-variate $c$. The values $p$ and $c$ do not necessarily need to reflect the exact value of the payoff, provided it is trivial to derive in software. For example, the value of $p$ for an arithmetic Asian option can be calculated in hardware as:

$$
p^{\prime}=\max \left(0, a_{T}-S_{T}(T+1)\right)
$$

rather than

$$
p=\max \left(0, \frac{a_{T}}{T+1}-S_{T}\right)
$$

since $S_{T}(T+1)$ can be pre-calculated, and the averaging (division by $T$ ) can also be applied by post-normalising in software. Because it takes at least $T$ steps to generate each path, in most cycles a kernel will not produce a value. In these empty cycles, the kernel will produce the pair $(0,0)$, in order to make the reduction tree simpler.

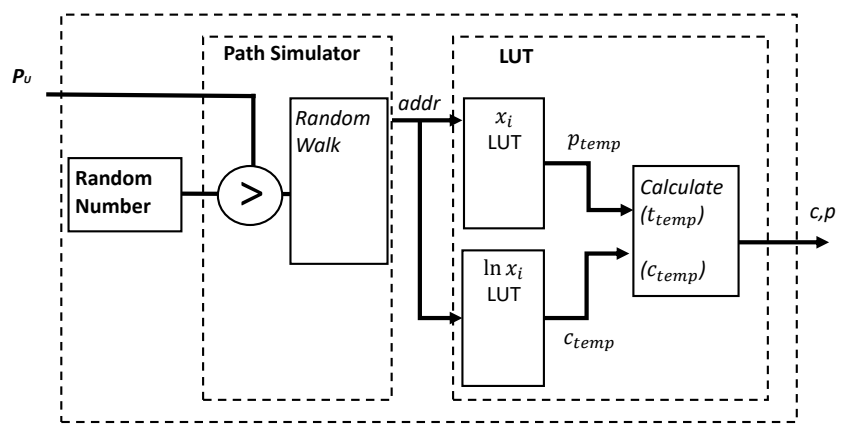

Fig. 11. The kernel diagram of the FPGA architecture

The binomial kernel is broken down into three entities, showing in Figure 11: A uniform random number generator; a path simulator; and a Look Up Table (LUT) containing the discrete space values of the random walk.

Random Number Generator : The random number generator used by the system is a 128-bit Xor-Shift[Marsaglia 2003]. A Xor-Shift random number generator is a 
pseudo-random number generator that requires very little hardware because it generates the next random number as an exclusive-or between a seed value and a bit-shifted version of the previous output. An important technical problem is to ensure that each kernel generates a different random stream, even though they all use the same RNG, is solved by ensuring that each kernel gets a different initial seed state. If we assume that we run for at most $2^{63}$ cycles, the 128-bit state means that we have $2^{64}$ distinct random streams that could be generated. The birthday paradox then shows that even if we have $2^{16}$ kernels, the probability of any two kernels using the same random numbers is less than $10^{-10}$.

Path Simulator : Internally, the random path simulator is simply an address, which is incremented or decremented according to the if statement in Algorithm 2. This address is used to index into the discrete space values stored in the LUT, which gives the current value of the underlying. To prevent out-of-bounds accesses to the LUT, a limiter is used to bound the random walk address to $[0, A)$, where $A=$ is the number number of entries in the LUT.

There are three main potential implementations of limiters: Bounce-off, Looparound, or Stick limiter. As long as the limiter is rarely encountered, it should have little effect on the global calculation, but different limiters will still have different effects on the calculated option price. The Bounce-off limiter sets the option price $a_{t}$ to $a_{t-1}$ on the next clock cycle. Similarly, a Stick limiter fixes $a_{t}$ to $a_{\text {limit }}$ for the rest of the random walk loop until $t=T$. A Loop-around limiter essentially does nothing, so the next step in the walk is at the opposite end of the RAM: $a_{t+1}=a 0$ if $a_{t}=A$ or $a_{t+1}=a A$ if $a_{t}=0$. For this paper, the exact effects of different limiters was not explored because practically the probability of reaching the limiter can be made negligible, as discussed later in Section 7. As a result this paper used a Bounce-off limiter, as it requires the least amount of hardware resources.

Look Up Table : The LUT can be thought of as a an $A \times W$ bit wide ROM for mapping the spatial index of the underlying to the pre-calculated discrete values associated with that index. For standard options, the values of $x_{i}$ will be tabulated and used in the payoff, as well as to calculate the arithmetic sum. For the geometric Asian controlvariate we also need to calculate the geometric mean of the path, which is implemented in another ROM containing the values of $\log x_{i}$, indexed by the same spatial variable. The geometric control variate is then generated as a summation:

$$
\begin{aligned}
\text { GeoMean } & =\left[\prod_{i=1}^{T} x_{i}\right]^{\frac{1}{T}} \\
& =\exp \left(\frac{1}{T} \sum_{i=1}^{T} \log \left(x_{i}\right)\right)
\end{aligned}
$$

Because the $e^{\hat{x} / T}$ calculation only happens once at the end of the path, it can be removed from the kernel, and absorbed into the accumulator, significantly reducing the size of each kernel.

A single physical RAM can be shared between two kernels by taking advantage of the even-odd properties of a binomial random walk. This optimization refers to the fact that it is impossible for two consecutive steps in a random walk to return to the same index in the LUT: a walk can only go up or down in the LUT, but never remains the same. Hence, the LUT can be divided into even and odd ROMs, and a second kernel can also be given access to the even and odd ROMs in alternate cycles. 

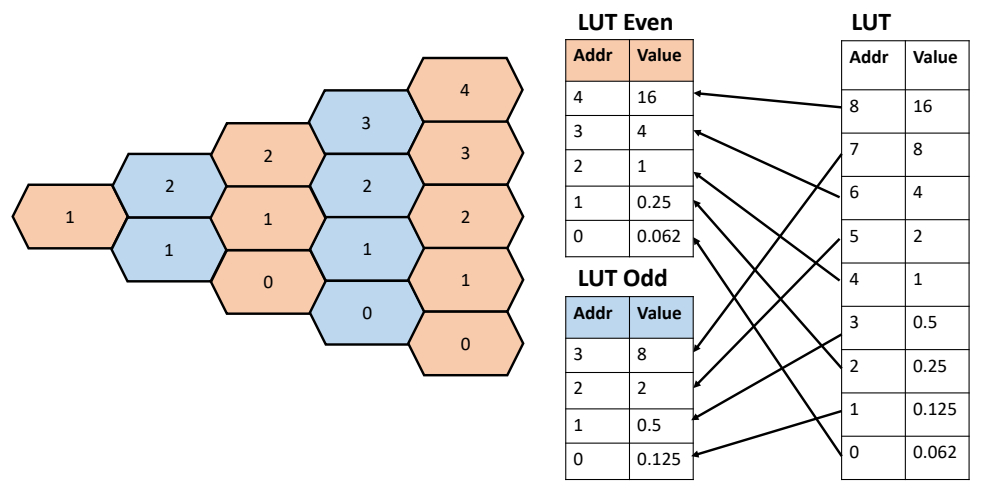

Fig. 12. A binomial random walk can be mapped to an even and odd LUT where neither walk overlaps.

Thus, two kernels can use the same overall LUT by splitting the LUT into two different even and odd ROMs, and four kernels can use the same LUT by accessing the dual ports on the physical ROMs, as shown in Figure 12, with the resulting hardware architecture shown in Figure 13. Note that the random number, path simulator and LUT logic is the same as before. The state machine is a toggle that ensures that the address variable is alternatively accessing different ROMs at each clock cycle. The merge element multiplexes between the two, and relies on the fact that only one of the kernels can produce a value in a given cycle.

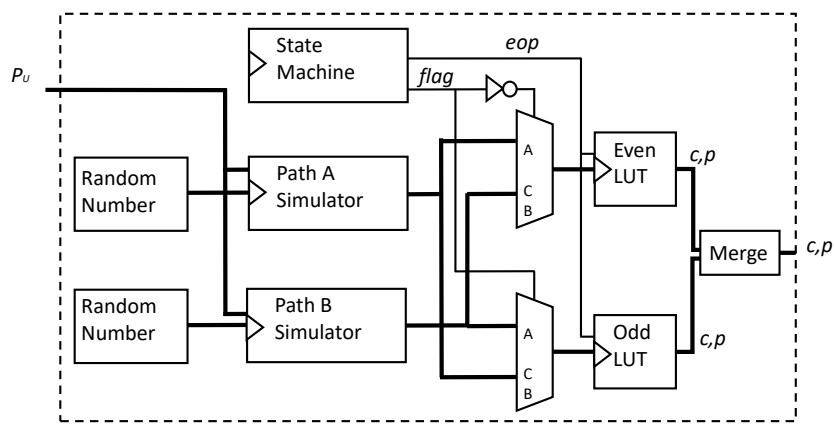

Fig. 13. The kernel diagram of the FPGA architecture whereby two path simulators share the resources of a single RAM element.

\subsection{Reduction Tree}

The reduction tree is a binary tree of mergers, with each merger able to combine two streams of $(p, c)$ payoff pairs. Because the kernels only produce non-zero $(c, p)$ pairs at the end of each path, we can implement the mergers with bit-wise $o r$, as long as we can guarantee that each merger input produces outputs in different cycles. We can ensure this by observing that each kernel differs only in the random number seed, and apart from that has exactly the same state machine. As a consequence, the state machines of 
the kernels can be started in a staggered fashion, ensuring that if kernel $k_{1}$ produces an output in cycle $i$, then kernel $k_{2}$ will produce an output at cycle $i+1$, kernel $k+3$ at cycle $i+2$, and so on. Only if we have $T$ or more cycles will we no longer be able to merge, and a new merger tree (and accumulator) is needed.

The reduction tree needs to be able to handle thousands of kernel instances, so it must be be scalable, without any long critical paths or global wiring. The hierarchical and statically scheduled nature of the tree means that we can handle extremely large fan-ins, simply by adding pipelining registers in each node of the tree. The extra $\log _{2}(N)$ latency is irrelevant to the overall throughput, but means that the global reduction is highly routable, and so can achieve high clock rates.

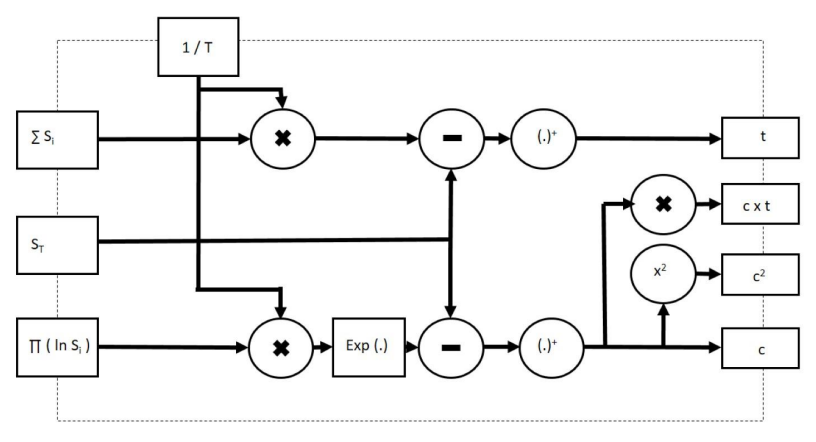

Fig. 14. Control-variate pre-processor

\subsection{Accumulator}

The final hardware stage is responsible for implementing any post-processing of the option payoff, and then for accumulating the control variate statistics. Only one accumulator is needed on each FPGA, provided the following two conditions hold:

- The steps per walk is greater than the number of kernels placed on the device $(N \leq$ $T)$.

- The output of each kernel is synchronized such that the results of one random walk enters the wrapper entity pipeline per clock cycle.

If $N>T$ we would need two accumulators, fed by two different trees, but for contemporary FPGA sizes and useful values of $T$ this has not been the case so far. As a consequence, the size and complexity of the accumulator is relatively unimportant, compared to the size of the kernels, so we can use area-intensive operators such as floating-point units and exponential units.

Figure 14 shows the post-processing needed for an arithmetic Asian with a geometric Asian control-variate. The multiplication by $1 / T$ and exponential are parts of the option payoff that have been moved from each of the $N$ kernels into the single accumulator, in order to reduce kernel size. The subtractors implement the $\max (0, x)$ payoff for the arithmetic and geometric options, and then the correlation measures needed for control-variate correction are calculated. The final $\left(p, c p, c^{2}, c\right)$ tuple is then accumulated, so that $E(p), E(c p), E\left(c^{2}\right), E(c)$ can be calculated in software. To remain efficient, the post-processing is done in single-precision floating point on the outputs of the reduction tree. 


\section{RESTRICTING LUT SIZE}

A key goal of the binomial approach is to have as many kernels as possible, which means they must be as small as possible. However, the results in Section 5 established that we may need to support $T$ up to $10^{4}$, which would require a LUT with $2 \times 10^{4}$ entries. Even with the ROM sharing approach described previously, this means that each kernel needs access to a ROM with $10 \mathrm{~K}$ entries. Contemporary block-RAMs are up to $1 \mathrm{~K}$ entries in length, so the need for 10 block-RAMs per kernel would mean that a lack of block RAMs severely limits scalability. If we can determine that the LUT entries will not overflow, then the ROM size can be limited to allow for one block RAM per kernel, enabling much higher $\mathrm{N}$.

We can achieve this by allowing for the possibility of overflowing the LUT, which means taking more steps in a random walk than is supported by the physical ROM size, and hitting the limiter. For example, 256 steps in a random walk can take 512 discrete possible values, and requires $A=512$. Taking more than 256 steps means there are multiple possible random walk paths whereby the path attempts to access bounds outside the LUT. Out-of-bounds addresses are bound by the limiter mentioned in random path simulator, and potentially cause bias in the results if they happen often.
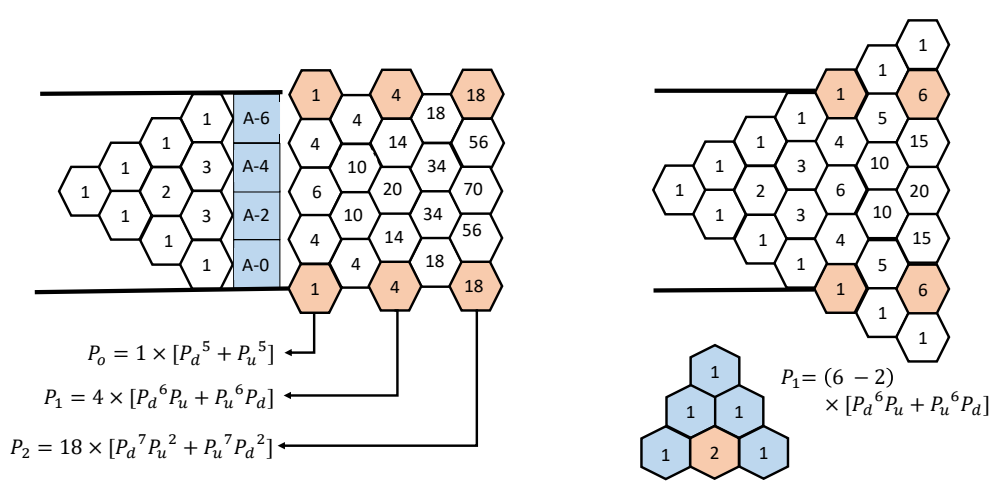

Fig. 15. The combinations of a binomial random walk with a limiter is defined by the pascals triangle less the paths through the upper or bottom addresses of the LUT.

The number of combinations of unique path that results in overflow for a given step $t$ and a LUT size $A$ can be calculated by the binomial coefficient at $\operatorname{Bin}(t, A)$, minus the combinations that are a result of paths that have already overflowed:

$$
L_{t}= \begin{cases}\left(\begin{array}{c}
t+A \\
s
\end{array}\right)-\sum_{i=1}^{\frac{t+1}{2}}\left(\begin{array}{c}
2 i \\
i
\end{array}\right) & t=\text { Odd } \\
0 & t=\text { Even }\end{cases}
$$

Note that a direct consequence of the binary walk is that only alternating steps in the walk can lead to overflowing the LUT - if a walk is at the limit of the LUT (address 0 or $A$ ), it can either overflow the LUT in the next step or revert to 1 or $(A-1)$. Figure 15 gives a graphical interpretation of the problem.

The first possibility of overflow occurs from a walk going only up or only down, so the probability of an overflow occurring for $t \leq A$ will be $P(o v \mid t \leq A)=0$, and the first possibility at $t=A+$ is $P(o v \mid t=A+1)=P_{u}^{A+1}+P_{d}^{A+1}$. The only way for a 
path to reach the bounds in the next possible overflow position, $t=A+3$, requires the walk to go down then up once, so the probability of overflow for $t=A+3$ will be $P(o v \mid t=A+3)=P_{u}^{A+2} P_{d}+P_{u} P_{d}^{A+2}$. Hence, a generalization of the probability of overflow at step $t$ is captured as:

$$
P(\text { ov } \mid t=x)= \begin{cases}0 & t \leq A \\ F_{t}\left(P_{u}^{A+(t-A+1) / 2} P_{d}^{(t-A+1) / 2}+P_{u}^{A+(t-A+1) / 2} P_{d}^{(t-A+1) / 2}\right) & \operatorname{odd}(t-A) \\ 0 & \operatorname{even}(t-A)\end{cases}
$$

Thus the total probability of overflow for a tree size of $T$ will be the cumulative distribution function of the probabilities $C D F(t=T)=\sum_{i=1}^{t} P(o v \mid t=i)$. From inspection, we can see that this will result in an extremely low probability of overflow, as long as $P_{U}$ is very close to 0.5 - in such cases the value of $P_{U}^{t}$ and $P_{D}^{t}$ decay rapidly, producing negligible probability of overflow.

Since the tuple $u, d, P_{u}$ is a function of $\delta t$, the value of $P_{u}$ and $P_{d}$ of a binomial pricing model is altered by changing the number of steps in the random walk. Figure 16 shows the minimum number of steps required to maintain the constraint that $\left|P_{u}-0.5\right|<0.01$, which ensures that the binomial tree is well balanced. Only in extreme cases (low rate of returns with high volatility options, or high rate of returns with low volatility options) is a large number of steps required before the probabilities balance out. The "envelope" shape is because in half of the values explored, more steps are needed to constrain the probability to under $51 \%$, and in the other half of the envelope more steps are needed to constrain the probability to above $49 \%$. Where the interest rates and the volatility effects cancel each other out is the canyon seen on the surface.

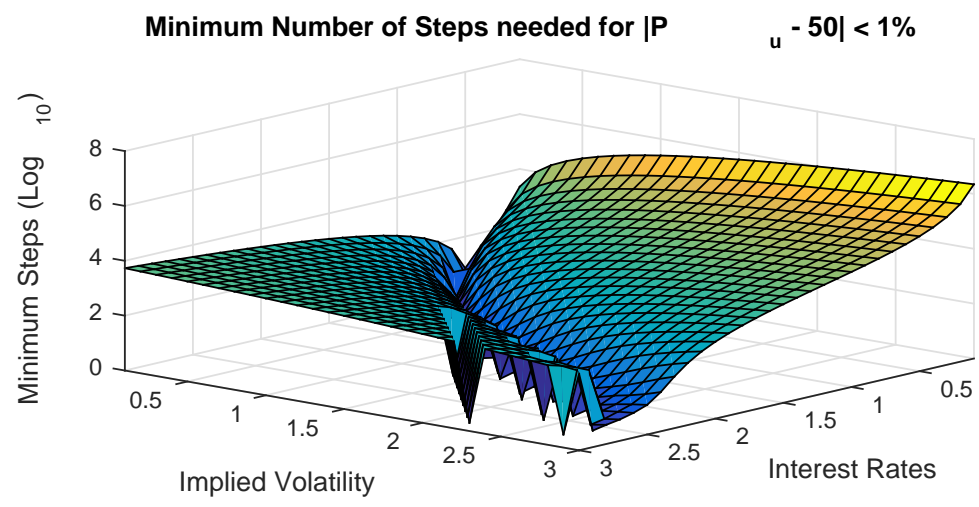

Fig. 16. The number of steps required to maintain $P_{u} 1 \%$ from $50 \%$ under different realistic market conditions.

Empirically calculating the value of $P_{u}$ for any implied volatility ranging from $0 \rightarrow$ $300 \%$, and return rates ranging from $0 \rightarrow 300 \%$, it can be shown that the probability $P_{u}$ is constrained to $[0.49,0.51]$ whenever the tree size is greater than $\approx\left(10^{7}\right)$. Notice that this constrains the system to $P_{U}=0.51$ when the volatility is 3 and the return rate is 0 . Less extreme market conditions and volatilities result in $P_{u}$ much closer to 0.5.

Additionally overflow in the LUT does not necessarily mean that the estimation from the Monte Carlo lattice is completely incorrect - the estimation is simply bounded by the upper region of the LUT. In terms of Asian option pricing, the error is approximately $\left(u^{A+1}-u^{A}\right) / T$ for the upper region of the LUT, and $\left(d^{A+1}-d^{A}\right) / T$ for the lower 
region of the LUT for each time the LUT bound is hit. Notice that due to the exponential scaling, the error as a result of the lower region of the LUT is $\approx 0$ for LUT size 512 . For look-back options this simply imposes a limit to the maximum or minimum return value of the Monte Carlo pricing. As such, the error introduced is the difference between the true maximum value of an unrestrained binomial walk and the limits of the LUT. For a barrier option, this imposes a system limitation - If the barrier is above the maximum value expressed in the LUT, then this architecture is no longer able to price the barrier option at all. However, in both the look-back and barrier option case, it is assumed that the market is pricing an interesting option, such that there is no obvious arbitrage on the option value. Nevertheless, this ensures that the architecture can keep the overflow error relatively low regardless of characteristics of the underlining.

It is important to recognize the trade-off between decreasing the size of the LUT and the accuracy of the result. While overflowing the LUT does introduce error to the system, the probability of error can be kept sufficiently small by manipulating the step size. Reducing the size of the LUT means more kernels can be packed into the FPGA, and hence more random walks can be performed concurrently. In an empirical Monte Carlo investigation, we considered the evaluation of a an Asian option with a table size of 512, looking at the error reduction as the size increases versus the error increase caused by the overflowing LUT. It was found that for tree sizes up to 5 billion the error due to overflow was still on the order of $10^{-12}$, while the reduction in error was around $10^{-7}$ for the option parameters specified. Note that these results were taken with a fixed iteration size of $K 100,000$ (simply to save computing time). In reality the optimal ratio will choose much larger $K$ and smaller $T$. In conclusion, in exchange for accepting a small probability of overflowing the LUT, an FPGA implementation is able to provide large $T$ while choosing $A<<T$.

\section{GAUSSIAN KERNEL}

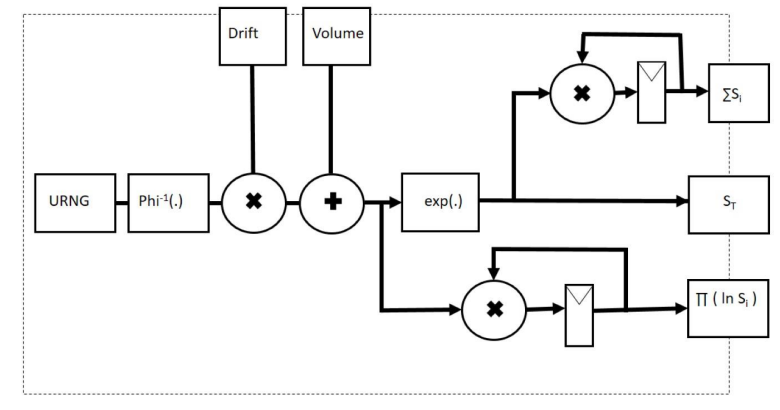

Fig. 17. The FPGA architecture of the Gaussian kernel that was implemented.

In order to compare the binomial approach with the Gaussian approach, a reference Gaussian implementation is needed. Previous work on control-variate Asian optons used much older FPGAs and floating-point IP [Tse et al. 2010], so we re-implemented their Gaussian architecture using modern tools Vivado HLS and floating-point cores. The fundamental simulation step is:

$$
\begin{aligned}
S_{i+1} & =S_{i} e^{D+V \times \operatorname{grand}()} \\
V & =\sigma \sqrt{\delta t} \quad D=\left(r-0.5 \sigma^{2}\right) \delta t
\end{aligned}
$$


The terms that are constant for each step in the walk are $D$ and $V$, which are precalculated in software. The core was designed to fit with the proposed reduction tree and accumulator, so only the kernels are changed.

All units in the architecture are single-precision floating-point, and pipelined, so the feedback loops through the multiplier take multiple cycles. As suggested in [Thomas et al. 2007], the C-Slow approach is used to accommodate this delay, resulting in multiple options flowing through the kernel at once. Notice that even by extracting all the constants calculated in the random walk, the Gaussian random number generator, exponential, adders, and multiplier blocks are all computationally intensive blocks required in every single kernel.

\section{EVALUATION}

Table III. The Resource Usage of a single kernel for the binomial and Gaussian models

\begin{tabular}{|l|l|l|l|l|l|l|}
\hline & Control Variate & BRAM & DSP & FF & LUT & MHz \\
\hline \hline Binomial & None & 0.5 & 0 & 326 & 149 & 300 \\
& European & 0.5 & 0 & 390 & 149 & 300 \\
& Geometric & 1 & 0 & 370 & 233 & 300 \\
\hline \hline Gaussian & None & 6 & 19 & 2001 & 3301 & 300 \\
& European & 6 & 21 & 2340 & 3638 & 300 \\
& Geometric & 6 & 25 & 3011 & 4311 & 300 \\
\hline \hline Combiner & & 0 & 31 & 3193 & 3906 & 300 \\
\hline
\end{tabular}

Table III displays the resource usage of both Gaussian and binomial Asian option models achieved and synthesized for a Xilinx UltraScale xcvu065-ffvc1517-3-e. The Asian option results are displayed because they are the biggest configuration for both the binomial and Gaussian design. All results are post place and route, with a clock constraint set at $300 \mathrm{Mhz}$. The place and route operations were performed using Vivado HLS 2016.4. The balance between DSP's and logic was manually tuned for the Gaussian model. The number of kernels $(n)$ placed on the device was defined by increasing $n$ until the timing constraints were no longer met. The devices investigated were Xilinx UltraScale XCVU065-FFVC1513-3-e (A), XCVU125-FLVA2104-3-e (B), XCVU160FLBG2104-2-e (C), XCVU190-FLGA2577-3-e (D), XCVU440-FLGA2892-3-e (E). These were intentionally chosen to give a good range of different FPGA sizes, BRAMs and DSP blocks.

Table IV outlines for each FPGA how many kernels were placed and routed for each FPGA. The multiplier improvement column indicates the multiple of more binomial kernels that can fit on a given device compared to the Gaussian model.

Table IV. The various number of kernels that were placed in the FPGA's

\begin{tabular}{|l|l|l|l|l|l|l|l|l|l|l|l|}
\hline Model & FPGA & BRAM & DSP & FF $\left(10^{3}\right)$ & LUT $\left(10^{3}\right)$ & \multicolumn{2}{|c|}{ No CV } & \multicolumn{2}{|c|}{ European CV } & Geometric CV \\
\hline \hline Gaussian & XCVU065 & 1260 & 600 & 35.8 & 71.6 & 26 & 1 & 24 & 1 & 20 & 1 \\
& XCVU125 & 2520 & 1200 & 71.6 & 143.2 & 55 & 1 & 50 & 1 & 42 & 1 \\
& XCVU160 & 3276 & 3276 & 92.6 & 185.3 & 153 & 1 & 139 & 1 & 116 & 1 \\
& XCVU190 & 3780 & 1800 & 107.4 & 214.8 & 83 & 1 & 75 & 1 & 63 & 1 \\
& XCVU440 & 2520 & 2880 & 253.3 & 506.6 & 134 & 1 & 122 & 1 & 102 & 1 \\
\hline \hline Binomial & XCVU065 & 1260 & 600 & 35.8 & 71.6 & 1968 & 75.69 & 1645 & 68.54 & 1134 & 56.70 \\
& XCVU125 & 2520 & 1200 & 71.6 & 143.2 & 3945 & 71.73 & 3297 & 65.94 & 2268 & 54.00 \\
& XCVU160 & 3276 & 3276 & 92.6 & 185.3 & 5106 & 33.37 & 4268 & 30.71 & 2948 & 25.41 \\
& XCVU190 & 3780 & 1800 & 107.4 & 214.8 & 5922 & 71.35 & 4950 & 66.00 & 3402 & 54.00 \\
& XCVU440 & 2520 & 2880 & 253.3 & 506.6 & 4536 & 33.85 & 4536 & 37.18 & 2268 & 22.24 \\
\hline
\end{tabular}


Figure 18 shows the trade-off between accuracy and solution time for both the current Gaussian FPGA implementation and the novel binomial method. As seen, the calculation frontier has been pushed forward with the novel design, and for all KT sizes there exists a KT combination for which the binomial model is clearly superior. It is important to note that there are combinations of the KT space such that the Gaussian model will perform better - this is further reason to why analysis of the KT space is crucial in the implementation. The results show that, given a constant error tolerance, the binomial method has a $50 \times$ average speed up compared to existing solutions on the FPGA, without any loss in accuracy, as illustrated by the horizontal black line in Figure 18. This is expected since there are roughly $50 \times$ as many kernels on the device and binomial and Gaussian kernels have similar KT properties. However, since the accuracy of a Monte Carlo simulation has a $\mathbb{O}(1 / \sqrt{x})$ relationship to the number of simulations performed, given a fixed time the binomial model is only $3.8 \times$ more accurate than using a Gaussian model (The vertical black line in figure 18).

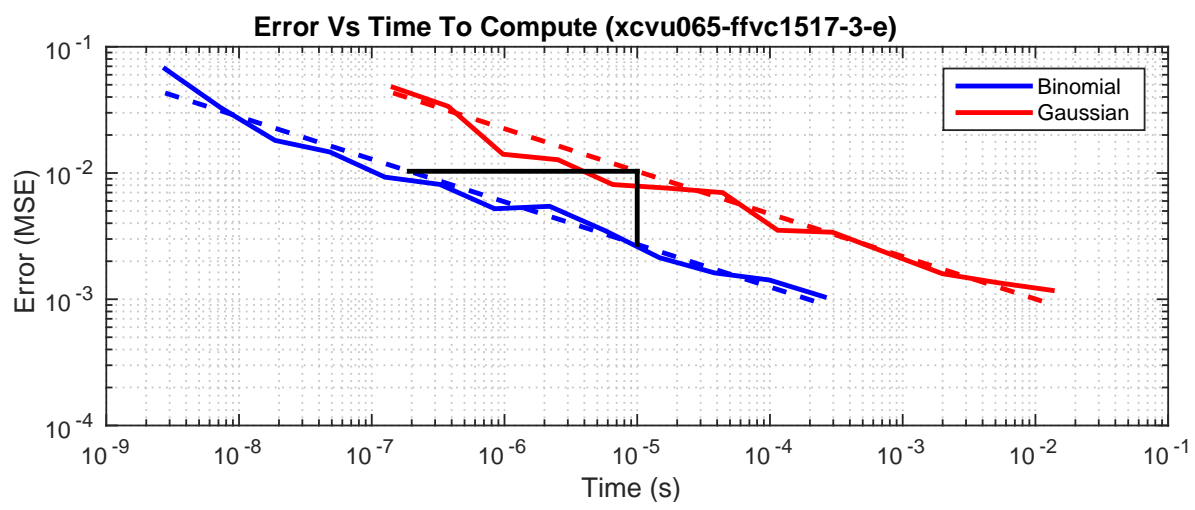

Fig. 18. The speed-vs-accuracy frontier comparisons between a Gaussian and binomial model

\section{CONCLUSION}

This paper proposes an alternative solution to the traditional Gaussian random walk for Monte Carlo pricing on FPGAs. The key idea is that if we discretise the random walk in both time and space, we can greatly reduce the area per simulator, and consequentially increase the number of simulations per second. To investigate this idea, we first establish that the binomial walk provides the same error performance as the Gaussian for the same number of time steps. In particular, we showed that the KT space of a Brownian motion walk and a binomial random walk is identical for the exotic options investigated, so both models can be used interchangeably. An architecture for implementing the simulation in an FPGA is then presented, showing that with careful optimisation the binomial simulation kernels can be reduced to a few hundred LUTs and one block RAM, compared to Gaussian kernels requiring thousands of LUTs and many DSP blocks.

The overall results show that the binomial Monte-Carlo approach is substantially better, allowing up $50 \times$ more evaluation cores to be placed in one FPGA, when compared to existing approaches. This results in either a $50 \times$ speed-up for the same accuracy, or a $4 \times$ reduction in error for the same time budget. Because the binomial technique also works with variance reduction such as control variates, this presents a large improvement over existing methods for exotic option evaluation in FPGAs. 
Future research includes investigating GPU implementations of the binomialforward Monte Carlo model, and supporting binomial multi-level Monte Carlo methods.

\section{REFERENCES}

HT Anson, David B Thomas, Kuen Hung Tsoi, and Wayne Luk. 2010. Reconfigurable control variate montecarlo designs for pricing exotic options. In Field Programmable Logic and Applications (FPL), 2010 International Conference on. IEEE, 364-367.

F. Black and M. S. Scholes. 1973. The Pricing of Options and Corporate Liabilities. Journal of Political Economics 81 (1973), 637-659.

Wissem Boughamoura and Faouzi Trabelsi. 2011. Variance reduction with control variate for pricing Asian options in a geometric levy model. IAENG International Journal of Applied Mathematics 41, 4 (2011), 320-329.

Phelim P Boyle. 1977. Options: A monte carlo approach. Journal of financial economics 4, 3 (1977), 323-338.

Antonio Camara. 2009. The Black-Scholes Legacy: Closed-Form Option Pricing Models. In Financial Derivatives. John Wiley and Sons, Inc., 387-404. DOI : http://dx.doi.org/10.1002/9781118266403.ch27

John C Cox, Stephen A Ross, and Mark Rubinstein. 1979. Option pricing: A simplified approach. Journal of financial Economics 7, 3 (1979), 229-263.

Christian De Schryver, Ivan Shcherbakov, Frank Kienle, Norbert Wehn, Henning Marxen, Anton Kostiuk, and Ralf Korn. 2011. An energy efficient FPGA accelerator for Monte Carlo option pricing with the Heston model. In Reconfigurable Computing and FPGAs (ReConFig). IEEE, 468-474.

Christian de Schryver, Pedro Torruella, and Norbert Wehn. 2013. A multi-level Monte Carlo FPGA accelerator for option pricing in the Heston model. In Proceedings of the Conference on Design, Automation and Test in Europe. EDA Consortium, 248-253.

P. Echeverria, M. Lopez-Vallejo, and J. M. Pesquero. 2008. Variance reduction techniques for Monte Carlo simulations. A parameterizable FPGA approach. In Int. Conf. on Electronics, Circuits and Systems. 1296-1299.

Michael C Fu, Dilip B Madan, and Tong Wang. 1999. Pricing continuous Asian options: a comparison of Monte Carlo and Laplace transform inversion methods. Journal of Computational Finance 2, 2 (1999), $49-74$.

Michael B Giles. 2008. Multilevel monte carlo path simulation. Operations Research 56, 3 (2008), 607-617.

Michael B. Giles. 2015. Multilevel Monte Carlo methods. In Acta Numerica, Volume 24. Cambridge University Press, 259-.

Steven L Heston. 1993. A closed-form solution for options with stochastic volatility with applications to bond and currency options. Review of financial studies 6, 2 (1993), 327-343.

Ralf Korn and Stefanie Müller. 2010. Binomial Trees in Option Pricing-History, Practical Applications and Recent Developments. Physica-Verlag HD, Heidelberg, 59-77. DOI : http://dx.doi.org/10.1007/978-3-7908-2598-5_3

George Marsaglia. 2003. Xorshift RNGs. Journal of Statistical Software 8, 14 (2003), 1-6.

Rahul Sridharan, Gregg Cooke, Kenneth Hill, Herman Lam, and Alan George. 2012. FPGA-Based reconfigurable computing for pricing multi-asset barrier options. In Application Accelerators in High Performance Computing (SAAHPC), 2012 Symposium on. IEEE, 34-43.

David B. Thomas, Jacob A. Bower, and Wayne Luk. 2007. Automatic Generation and Optimisation of Reconfigurable Financial Monte-Carlo Simulations. In Proc. ASAP. 168-173.

Anson HT Tse, David B Thomas, Kuen Hung Tsoi, and Wayne Luk. 2010. Efficient reconfigurable design for pricing asian options. ACM SIGARCH Computer Architecture News 38, 4 (2010), 14-20.

G. L. Zhang, P. H. W. Leong, C. H. Ho, K. H. Tsoi, D-U. Lee, R. C. C. Cheung, and W. Luk. 2005. Reconfigurable Acceleration for Monte Carlo based Financial Simulation. In Proc. FPT. 215-224. 